\title{
GROWTH-OPTIMAL STRATEGIES WITH QUADRATIC FRICTION OVER FINITE-TIME INVESTMENT HORIZONS
}

\author{
ERIK AURELL \\ Swedish Institute of Computer Science \\ SICS Box 1263 SE-16429, Kista, Sweden \\ erik.aurell@sics.se \\ and \\ Department of Numerical Analyis and Computer Science \\ KTH-Royal Institute of Technology, SE-100 44 Stockholm, Sweden \\ PAOLO MURATORE-GINANNESCHI \\ Departments of Mathematics \\ P.O. Box 4 FIN-00014, University of Helsinki, Finland \\ paolo.muratore-ginanneschi@helsinki.fi
}

\begin{abstract}
We investigate the growth optimal strategy over a finite time horizon for a stock and bond portfolio in an analytically solvable multiplicative Markovian market model. We show that the optimal strategy consists in holding the amount of capital invested in stocks within an interval around an ideal optimal investment. The size of the holding interval is determined by the intensity of the transaction costs and the time horizon.
\end{abstract}

\section{Introduction}

An idealised model of investment is a sequence of gambles where the speculator chooses at each time step her position. The game is multiplicative if the pay-off is proportional to the capital, and it is Markov if the new capital and new position depend parametrically only on the previous state. The relevant issue consists of determining which strategy the speculator should pursue, which in general depends on the preferences of the individual investor. A reasonable choice is to 
assume that the investor wishes to maximise the growth of her capital. In this paper we will investigate such strategies for finite-time investment horizons. Another interpretation is then that we will be looking at investments in a class of quadratic utility functions, where, for definiteness, we take certain values of the parameters, which are then those that correspond to growth-optimal investment. The main novelty is that we include transaction costs. With the special functional form of the costs studies here, the investment problem can be solved analytically by mapping to an example in quantum mechanics.

Growth optimal criteria for multiplicative Markov process were first investigated by Kelly in the context of information theory [14]. Criticisms from the viewpoint of utility theory appeared later in the economic literature. For recent reviews the reader is referred to refs. [13, 15, 2]. A related but separate question is what to do if the laws of the gambles is unknown, but has to be deduced from observing price history (or other information). A widely recognised procedure is then the "Universal portfolios" [6, 7], which can be considered growth-optimal strategies, using continuously updated guesses for the parameters of the model. Growth-optimal criteria are therefore robust to the market participnats lacking knowledge of the statistical laws of the market. This makes them interesting also over finite-time horizons.

In a previous contribution [3] we derived from a discrete multiplicative Markov model the continuum limit dynamics of a stock and bond portfolio in the presence of linear trading costs, i.e. proportional to the absolute value of the capital moved by the investor to balance her portfolio. Our main result can be summarised by saying that on an infinite time horizon the investment optimal strategy consists of allowing the amount of capital invested in stocks to fluctuate freely within an interval around the value of the optimal investment in the absence of trading costs. The size of the holding interval was shown to depend non-analytically on an adimensional parameter measuring the intensity of the transaction costs. In [3] we took the existence of growth-optimal strategies over infinite time horizons for granted. This has recently been proven, and in a much more general setting [8]. A consequence of the result on the holding interval is that that for financially reasonable values of the parameters in the model, convergence to the full dynamical solution in the infinite time horizon limit may be surprisingly slow, of the order of years of trading. It is therefore natural to address the question of the time evolution of the growth optimal strategy over finite investment horizons. The question has relevance also in the perspective of deriving an option pricing procedure from growth optimal criteria.

In the present paper we tackle the problem of an optimal strategy in a market model where trading costs are described by a quadratic function of the fraction of capital invested in re-hedging the portfolio. One advantage of this model that it is mathematically simpler, since the costs are analytic. Indeed, we will show that the model can essentially be solved analytically, which is interesting in itself. From the financial side, one can compare with the market impact phenomenology of [9]. Suppose one first buys $w$ worth of shares, and sells off shares to recover the same amount $w$. By actively seeking a deal one is forced to buy high and sell low. The turn-around cost of the operation is then on average $w^{2} / \lambda$, under 
the assumption that $w$ is much less than the market depth $\lambda$. If one assumes that market depth grows proportionally to the total wealth of a typical investor in the market one recovers the model we study here. The analysis we will present can therefore, for example, be relevant to fairly large operators in a market, the actions of which move market prices, to some extent.

The paper is organised as follows. In section 2 we state the optimisation problem in the framework of the Hamilton-Jacobi-Bellman equation, well known in mathematical finance. In section 3 we show that the non-linear Hamilton-JacobiBellman equation governing the dynamics, in our example, is solvable in the small transaction costs limit by means of a multi-scale perturbation theory (see for example [10, 5], or [12], chapter 9). This is the main technical result of the paper, and reduces the non-linearity to a normal form. All higher order corrections can be computed from ancillary linear non-homogeneous equations. In section $\bigoplus$ we solve analytically the normal form of the non-linearity and compare the result with the numerical solution of the original Hamilton-Jacobi-Bellman equation. The approximation turns out to be very accurate for realistic values of the parameters in the model. The last section is devoted to a discussion of the results.

\section{HJB equation for trading costs}

The stock and bond trade dynamics is in the continuum limit governed by the system of stochastic differential equations

$$
\begin{aligned}
& d W_{t}=\left[\mu \rho_{t}-\gamma f^{2}\left(\rho_{t}, t\right)\right] W_{t} d t+\sigma \rho_{t} W_{t} d B_{t} \\
& d \rho_{t}=\left[f\left(\rho_{t}, t\right)+a\left(\rho_{t}\right)+\gamma \rho_{t} f^{2}\left(\rho_{t}, t\right)\right] d t+b\left(\rho_{t}\right) d B_{t}
\end{aligned}
$$

with

$$
\begin{aligned}
& a\left(\rho_{t}\right)=\rho_{t}\left(1-\rho_{t}\right)\left(\mu-\sigma^{2} \rho_{t}\right) \\
& b\left(\rho_{t}\right)=\sigma \rho_{t}\left(1-\rho_{t}\right)
\end{aligned}
$$

In the equations (11)-(2), $W_{t}$ is the total wealth of the speculator at time $t$, and $\rho_{t}$ is the fraction of the wealth held in stocks at time $t$. The stochastic control $f$ represents the action taken by the speculator at time $t$ to re-hedge her position in the market. The stochastic control is to be determined as a function of $\rho_{t}$ and $t$, by maximising the expectation value of the wealth growth:

$$
\lambda(x, t ; T)=E_{\rho_{t}=x} \ln \frac{W_{T}}{W_{t}}=E_{\rho_{t}=x} \int_{t}^{T} d s\left[\mu \rho_{s}-\frac{\sigma^{2}}{2} \rho_{s}^{2}-\gamma f^{2}\right]
$$

The expectation $E_{\rho_{t}=x}$ is conditional on the fraction in stock process $\rho_{t}$ having value $x$ at initial time $t$. The time difference $T-t$ is the time horizon of the speculator: the time period wherein she wants to optimise her position in the 
market. The optimisation is performed with respect to two conflicting effects. On one hand, the market fall-outs raise or lower the relative amount of invested wealth, motivating the investor to re-balance the portfolio. On the other, the rehedging carries trading costs. In this paper we model these as $\gamma f^{2}$, where $\gamma$ is some given positive valued constant. The derivation of the equations (11)-(2) from a discrete multiplicative Markov game is given in appendix.

For any stochastic control $f$ such that the system (11), (2) is well defined, the expectation value of the wealth growth must obey the dynamic programming equation

$$
\begin{aligned}
& \partial_{t} \lambda+\left[f+a+\gamma x f^{2}\right] \partial_{x} \lambda+\frac{b^{2}}{2} \partial_{x}^{2} \lambda+\mu x-\frac{\sigma^{2} x^{2}}{2}-\gamma f^{2}=0 \\
& \lambda(x, T ; T)=0
\end{aligned}
$$

The functional dependence of the drift $a$ and of the diffusion coefficient $b$ on $x$ is defined by (3) and has been omitted to streamline the notation. The optimisation problem is well defined only when the boundary conditions in $x$ are specified. The solution of the dynamic programming equation (5) has by construction the form of an average over the transition probability density of the stochastic process $\rho_{t}$. In the model considered here we can neglect capital borrowing and lending since (under proper conditions on the parameters) the process will never move to $\rho$ outside the interval $[0,1]$. The conservation of the probability measure then implies

$$
\left.\partial_{x} \lambda(x, t ; T)\right|_{x=0}=\left.\partial_{x} \lambda(x, t ; T)\right|_{x=1}=0
$$

which are adjoint to the reflecting boundary conditions imposed on the probability density.

The growth is stationary versus the control $f$ if

$$
\frac{\delta \lambda(., t)}{\delta f\left(., t^{\prime}\right)}=0
$$

For any finite $\gamma$, the extremum condition yields a relation between the stochastic control and the expected growth:

$$
(1+2 \gamma x f) \partial_{x} \lambda-2 \gamma f=0
$$

The extremum is a maximum for

$$
\Phi(f, \lambda)=\left[f+a+\gamma x f^{2}\right] \partial_{x} \lambda+\frac{b^{2}}{2} \partial_{x}^{2} \lambda+\mu x-\frac{\sigma^{2} x^{2}}{2}-\gamma f^{2}
$$

provided [16, 11]

$$
2 \gamma x \partial_{x} \lambda-2 \gamma<0
$$


Thus, the optimal stochastic control is

$$
f=\frac{\partial_{x} \lambda}{2 \gamma\left(1-x \partial_{x} \lambda\right)}
$$

The Hamilton-Jacobi-Bellman [16] equation governing the optimal dynamics is then

$$
\begin{aligned}
& \partial_{t} \lambda+a \partial_{x} \lambda+\frac{\left(\partial_{x} \lambda\right)^{2}}{4 \gamma\left(1-x \partial_{x} \lambda\right)}+\frac{b^{2}}{2} \partial_{x}^{2} \lambda+\mu x-\frac{\sigma^{2} x^{2}}{2}=0 \\
& \lambda(x, T ; T)=0 \\
& \left.\partial_{x} \lambda(x, t ; T)\right|_{x=0}=\left.\partial_{x} \lambda(x, t ; T)\right|_{x=1}=0
\end{aligned}
$$

This equation is time autonomous and therefore the solution can be sought in the form

$$
\lambda(x, t ; T) \equiv \lambda(x, T-t)
$$

In view of the ensuing analysis of the Hamilton-Jacobi-Bellman equation it is useful to identify the canonical dimensions of the quantities involved in the problem:

$$
\begin{array}{lll}
{[\lambda]=0} & {[x]=0} \\
{\left[\sigma^{2}\right]=[1 / t]} & {[\mu]=[1 / t]} \\
{[\gamma]=[t]} & {[f]=[1 / t]}
\end{array}
$$

A trading day can be assumed to define the time unit. Note that the dimensions in (14) are partially different from those given in [3] since the functional form of the friction is different.

\section{The transaction cost free limit and its leading or- der correction}

In the absence of transaction costs the speculator is free to take un-restrained actions to always keep the fraction allocated to stocks constant

$$
\rho^{\mathrm{opt}}=\frac{\mu}{\sigma^{2}}
$$

This gives [1], 16] the absolute value of the wealth growth:

$$
\left.\lambda(x, t ; T)\right|_{\gamma=0}=\frac{\mu^{2}}{2 \sigma^{2}}(T-t)
$$


In this paper we assume $\mu$ and $\sigma^{2}$ are such that the ratio (15) lies inside the interval $[0,1]$, see the appendix for discussion.

In the frame-work of the dynamics programming equation, the absence of trading costs renders (5) linear in the stochastic control $f$. The optimal growth is achieved by wielding a singular control strategy which restricts the support of the probability measure of $\rho_{t}$ only to the point $\rho^{\text {opt }}$. Thus, when trading costs are present but "small", it must be possible to seek to solve of the HamiltonJacobi-Bellman equation (12) by means of a perturbative expansion around the limit (16). In order to do that it is convenient to translate the origin of the $x$ coordinate according to

$$
x \rightarrow x+\frac{\mu}{\sigma^{2}}
$$

For any finite $\sigma^{2}$, the adimensional parameter measuring the intensity of trading costs is

$$
\epsilon=\sigma^{2} \gamma
$$

However, the perturbative expansion cannot be analytic in $\epsilon$. This can be argued a priori by observing that Oseledec' theorem [17] predicts for the asymptotic behaviour of the wealth growth

$$
\lim _{t \downarrow-\infty} \lambda(x, t ; T) \sim(T-t) \ell
$$

It is therefore natural to assume that the dynamics of the wealth growth $\lambda$ should involve two typical time scales. The first time scale should be $\ell^{-1}$ governing the asymptotic regime, while the second should describe the characteristic relaxation time to the asymptotic regime. Such considerations, together with the singular dependence of the Hamilton-Jacobi-Bellman equation (12) on $\gamma$, suggest the Ansatz

$$
\lambda(x, t ; T)=\epsilon \varphi\left(\frac{x}{\epsilon^{1 / 4}}, \frac{T-t}{\epsilon}, \frac{T-t}{\epsilon^{1 / 2}}\right)
$$

The first time dependence refers to the linear growth. The second describes the relaxation process and it is conjectured to satisfy in $\epsilon$ the characteristic time-spatial scaling relation of diffusion processes. From (20) it appears that small transaction cost expansion of $\lambda$ entails a multi-scale perturbation theory with times

$$
\begin{aligned}
r & :=\frac{T-t}{\epsilon} \\
s & :=\frac{T-t}{\epsilon^{1 / 2}}
\end{aligned}
$$

and consequently

$$
\partial_{t}=\frac{1}{\epsilon} \partial_{r}+\frac{1}{\epsilon^{1 / 2}} \partial_{s}
$$


The "spatial" rescaling

$$
y:=\frac{x}{\epsilon^{1 / 4}}
$$

finally yields for

$$
\varphi=\varphi(y, r, s)
$$

the equation

$$
\begin{aligned}
0= & \partial_{r} \varphi+\frac{\mu^{2}}{2 \sigma^{2}}+\epsilon^{1 / 2}\left[\partial_{s} \varphi+\frac{\sigma^{4}\left(\partial_{y} \varphi\right)^{2}}{4\left(\sigma^{2}+\epsilon^{3 / 4}\left(\mu+\epsilon^{1 / 4} y \sigma^{2}\right) \partial_{y} \varphi\right)}\right. \\
& \left.+\frac{\left(\mu+\epsilon^{1 / 4} y \sigma^{2}\right)^{2}\left(\mu+\left(\epsilon^{1 / 4} y-1\right) \sigma^{2}\right)^{2}}{2 \sigma^{6}} \partial_{y}^{2} \varphi-\frac{\sigma^{2} y^{2}}{2}\right] \\
& +\frac{\epsilon y\left(\mu+\epsilon^{1 / 4} y \sigma^{2}\right)\left(\mu+\left(\epsilon^{1 / 4} y-1\right) \sigma^{2}\right)}{\sigma^{2}} \partial_{y} \varphi
\end{aligned}
$$

The equation admits now an analytic expansion in powers of $\epsilon^{1 / 4}$ the solution whereof is amenable to the form of the series

$$
\varphi=\frac{\mu^{2} r}{2 \sigma^{2}}+\sum_{n=0}^{\infty} \epsilon^{n / 4} \varphi_{n}(y, s)
$$

The equation for $\varphi_{0}(y, s)$ is non-linear and provides the normal form of the nonlinearity involved in the Hamilton-Jacobi-Bellman problem (12):

$$
\partial_{s} \varphi_{0}+\frac{\sigma^{2}\left(\partial_{x} \varphi_{0}\right)^{2}}{4}+\frac{D^{2}}{2} \partial_{x}^{2} \varphi_{0}-\frac{\sigma^{2} y^{2}}{2}=0
$$

The effective "diffusion" constant $D^{2}$ in (27) is

$$
D^{2}=\sigma^{2}\left(\frac{\mu}{\sigma^{2}}\right)^{2}\left(1-\frac{\mu}{\sigma^{2}}\right)^{2}, \quad\left[D^{2}\right]=[1 / t]
$$

Terms of higher orders in the expansion (26) are obtained by solving linear nonhomogeneous equations. The first of them is for example provided by the solution of

$$
\begin{aligned}
& \partial_{s} \varphi_{1}+\frac{1}{2} \sigma^{2} \partial_{y} \varphi_{0} \partial_{y} \varphi_{1}+\frac{D^{2}}{2} \partial_{y}^{2} \varphi_{1} \\
& \quad+\frac{\left(2 \mu^{2} y \sigma^{2}\left(\mu-\sigma^{2}\right)+2 \mu y \sigma^{2}\left(\mu-\sigma^{2}\right)^{2}\right)}{2 \sigma^{6}} \partial_{y}^{2} \varphi_{0}=0
\end{aligned}
$$


The conclusion of the above analysis is that the leading order in the multi-scale expansion captures the effect of the non-linearity involved in the optimisation problem. Otherwise stated, within leading order in the small trading costs limit, equation (12) can be consistently replaced by the simpler model problem

$$
\begin{aligned}
& \partial_{t} \lambda+\frac{\left(\partial_{x} \lambda\right)^{2}}{4 \gamma}+\frac{D^{2}}{2} \partial_{x}^{2} \lambda+\frac{\mu^{2}}{2 \sigma^{2}}-\frac{\sigma^{2} x^{2}}{2}=0 \\
& \lambda(y, T ; T)=0 \\
& \left.\partial_{x} \lambda(x, t ; T)\right|_{x=-\frac{\mu}{\epsilon^{1 / 4} \sigma^{2}}}=\left.\partial_{x} \lambda(x, t ; T)\right|_{x=\frac{1}{\epsilon^{1 / 4}}\left(1-\frac{\mu}{\sigma^{2}}\right)}=0
\end{aligned}
$$

The model problem is the Hamilton-Jacobi-Bellman equation associated to the dynamic programming equation

$$
\partial_{t} \lambda+\sup _{f}\left\{f \partial_{x} \lambda+\frac{D^{2}}{2} \partial_{x}^{2} \lambda+\frac{\mu^{2}}{2 \sigma^{2}}-\frac{\sigma^{2} x^{2}}{2}-\gamma f^{2}\right\}=0
$$

The extremum conditions here become

$$
\begin{aligned}
& \partial_{x} \lambda-2 \gamma f=0 \\
& -2 \gamma<0
\end{aligned}
$$

stating that the solution is always a maximum. This fact guarantees that (30) provides the optimal capital growth for $\epsilon$ small enough.

\section{The logarithmic transform}

The model problem (30) can be mapped to a linear equation by the logarithmic transform

$$
\lambda(x, t ; T)=A \ln \psi(x, T-t)
$$

provided

$$
\psi(x, T-t)>0
$$

The initial condition for the function $\psi$ is

$$
\psi(x, 0)=1
$$

The value of the constant $A$ is fixed by imposing the cancellation of the non-linear term. Namely, by setting

$$
A=2 D^{2} \gamma, \quad[A]=0
$$


(31) is mapped into the imaginary time Schrödinger equation

$$
-\partial_{t} \psi+\frac{D^{2}}{2} \partial_{x}^{2} \psi+\frac{1}{2 D^{2} \gamma}\left[\frac{\mu^{2}}{2 \sigma^{2}}-\frac{\sigma^{2} x^{2}}{2}\right] \psi=0
$$

The equation further simplifies if the Neumann boundary conditions on a finite interval of length proportional to $\epsilon^{-1 / 4}$ are replaced by Dirichlet boundary conditions at infinity:

$$
\lim _{|x| \uparrow \infty} \psi(x, t)=0
$$

As a matter of fact, $(37)$ admits on $\mathbb{L}^{2}(\mathbb{R})$ the explicit solution (see for example appendix A.4 in [[18]):

$$
\psi(x, t)=e^{\frac{1}{2 D^{2} \gamma} \frac{\mu^{2} t}{2 \sigma^{2}}} \sum_{n=0} e^{-E_{2 n} t} \psi_{2 n}(x) \int_{\mathbb{R}} d y \psi_{2 n}(y)
$$

where

$$
\begin{array}{ll}
\psi_{n}(x)=\frac{1}{\sqrt{2^{n} n !}}\left(\frac{1}{\pi \tau D^{2}}\right)^{1 / 4} e^{-\frac{x^{2}}{2 \tau D^{2}}} H_{n}\left(\frac{x}{\sqrt{\tau D^{2}}}\right) \\
E_{n}=\frac{1}{\tau}\left(n+\frac{1}{2}\right), & \frac{1}{\tau}=\sqrt{\frac{\sigma^{2}}{2 \gamma}}
\end{array}
$$

for $H_{n}(\xi)$ the $n$-th Hermite polynomial. The series (39) is restricted to even values of $n$ since Hermite polynomials are of even/odd parity for even/odd $n$

$$
H_{n}(-\xi)=(-)^{n} H_{n}(\xi)
$$

The logarithmic transform is well defined on (39) due to the exponential decay of the coefficients in the series

$$
\frac{1}{\sqrt{2^{n} n !}}\left(\frac{1}{\pi \tau D^{2}}\right)^{1 / 4} \int_{\mathbb{R}} d y \psi_{2 n}(y)=\frac{(2 n-1) ! ! \sqrt{2}}{2^{n}(2 n) !}
$$

Hence, the average optimal growth of the capital as a function of the fraction initially invested in stocks $x$ on a time horizon $T-t$ is

$$
\begin{gathered}
\lambda(x, t ; T)=\left[\frac{\mu^{2}}{2 \sigma^{2}}-\frac{D^{2} \sigma^{2} \tau}{2}\right](T-t)-\frac{\sigma^{2} \tau x^{2}}{2}+D^{2} \sigma^{2} \tau^{2} \ln \sqrt{2} \\
+D^{2} \sigma^{2} \tau^{2} \ln \left\{\sum_{n=0}^{\infty} \frac{(2 n-1) ! ! e^{-2 n \frac{T-t}{\tau}}}{2^{n}(2 n) !} H_{2 n}\left(\frac{x}{\sqrt{\tau D^{2}}}\right)\right\}
\end{gathered}
$$




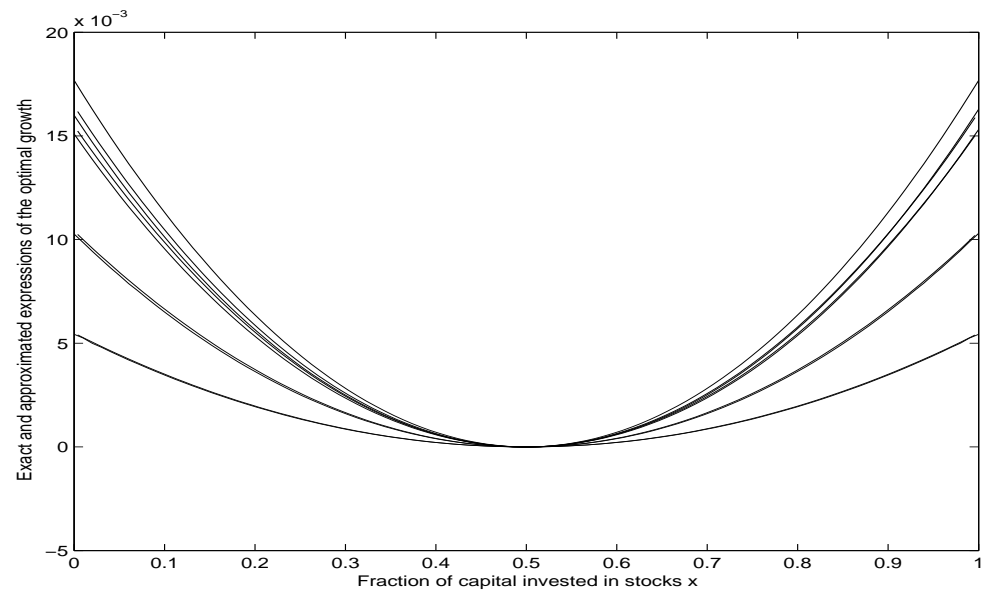

Figure 1: The control potential $2 \gamma V$ as defined in (46) for $\mu=\sigma^{2} / 2, \sigma=10^{-2}$ and $\gamma=10^{2}$ is plotted for time horizons of $T-t=500,1000,2000,2500$ days using both the exact solution of the Hamilton-Jacobi-Bellman equation (12) and the approximate solution (43). The characteristic decay time to the asymptotic regime is $\tau / 2 \sim 1000$ days. The innermost parabola is obtained from the asymptotic expression (47).

In agreement with Oseledec' theorem (19), convergence to a stationary state is exponentially fast with rate equal to

$$
\frac{\tau}{2}=\frac{1}{2} \sqrt{\frac{2 \gamma}{\sigma^{2}}} \equiv \frac{1}{\sigma^{2}} \sqrt{\frac{\epsilon}{2}}
$$

From the last identity it is also straightforward to verify the consistency of (43) with the multi-scale Ansatz (20). Finally, the time asymptotic form of the solution can be extracted directly from (30) as it was done in our previous paper [3] by using the information (19) provided by Oseledec' theorem.

\section{Discussion and conclusions}

The exact solution of the model problem (30) gives a qualitatively and for $\sigma^{2} \gamma$ small enough also quantitatively correct description of the investment strategy that a speculator should pursue in order to optimise her profits. The strategy is most conveniently summarised by looking at the potential $V$

$$
f(x, T-t)=-\partial_{x} V(x, T-t)
$$




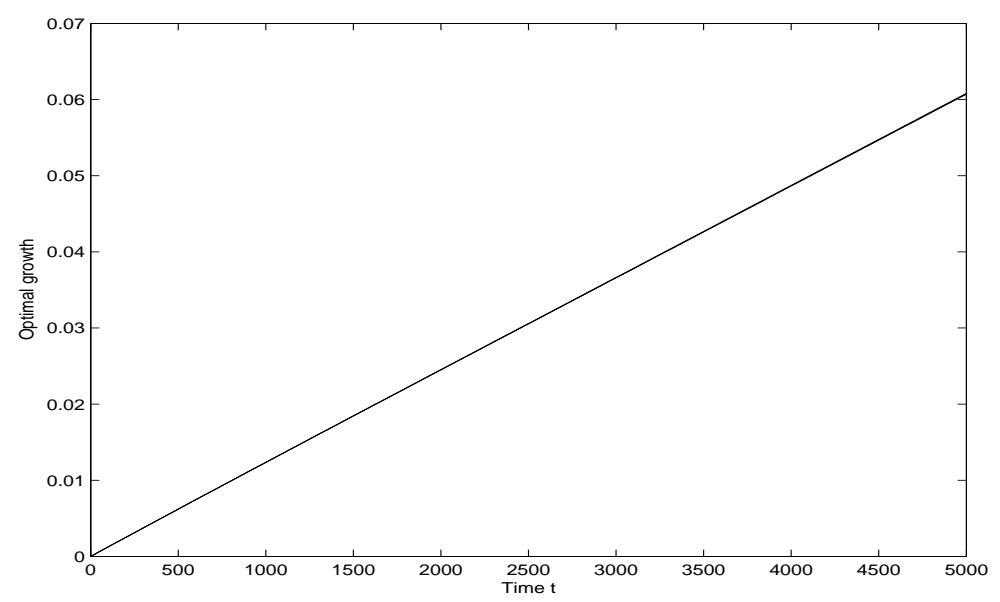

Figure 2: The exact and approximate optimal growth $\lambda(0, t)$ is plotted versus the time horizon for the same parameters used in (47). The two lines almost overlap.

associated to the optimal stochastic control. Within the same approximation leading to (30), the potential can be defined as

$$
V(x, T-t)=-\frac{\lambda(x, T-t)}{2 \gamma}+\frac{\lambda(0, T-t)}{2 \gamma}
$$

The behaviour in time of the potential is illustrated in figure 1. In the figure the same quantity (46) is also plotted when $\lambda$ is obtained from the solution of the exact Hamilton-Jacobi-Bellman equation (12). The resulting profiles are practically indistinguishable for financially reasonable choices of the parameters in the perturbative regime. The shape of the potential entails a fast decay of the probability density of the fraction of capital invested in stocks versus the deviation from the ideal optimum (15). The observation justifies a posteriori the use of Dirichlet boundary conditions in the solution of the model problem. As a matter of fact, the modification of the boundary conditions affects the average (4) only in a region where the probability density is practically equal to zero.

The potential gets steeper when time to investment horizon is long, tending asymptotically to a parabolic shape

$$
V_{\text {asympt. }}(x)=\frac{x^{2}}{2 \tau} \quad(T-t)>>\tau
$$

In this limit the speculator aims to always hold the invested fraction of capital in a finite interval around the optimal investment fraction $\rho^{\text {opt }}$ of (15). In the asymptotic regime the fraction of capital invested in stocks tends to an OrnsteinUhlenbeck process [卂] the invariant measure whereof having variance $D^{2} \tau / 2$. 
This latter quantity provides the typical size of the holding interval. When $T-t$ is on the order of $\tau$, or smaller, the solution is given by the complete expression (43), with corrections from the higher order terms in (26). The general behaviour of this process is a successively flatter potential leading to a larger holding interval, see Fig. 2.

The results of the present paper together with those of our previous contribution [3] support an "investment confinement" picture as growth optimal strategy for multiplicative Markov market models with trading costs. According to such picture, differences in the modeling of the trading costs are reflected only in the different non-analytic powers of $\epsilon$ on which the size of the holding interval depends. Differences in the modeling of transaction costs do not seem to affect the typical time scales governing the relaxation to the asymptotic growth state. Namely also in the case of quadratic market friction, a daily relative stock price fluctuation $\sigma^{2}$ of the order of $10^{-4}$ per cent with $\epsilon$ equal to 0.01 yields for decay rate the value of 700 days. The result is in agreement with the prediction of the dimensional analysis in [3].

\section{Acknowledgements}

The authors gratefully acknowledge discussions with A. Kupiainen. This work was supported by the Swedish Research Council through grant NFR I 510-930 (E.A.), and grant "hanke" nr. 39746 “päätös" nr. 68820 from the Finnish Academy of Science (P.M.G.).

\section{Appendix}

We present here a derivation of the continuum limit market dynamics slightly different than the one given in [3]. At time $t$ the wealth in stocks is, in units of the total wealth,

$$
W_{t}^{(\text {Stocks })}=\rho_{t} W_{t}
$$

The variation in one time step of the wealth in stocks occurs in consequence of

- the market fall-out $u_{t}$

- the action $\Delta \chi_{t}$ of the speculator who re-hedges her position in the market.

The fraction in stocks at time $t+1$ becomes

$$
W_{t+1}^{(\text {Stocks })}=\left[u_{t} \rho_{t}+\Delta \chi_{t}\right] W_{t}
$$

The total wealth at time $t+1$ is affected by the stock investment profits or losses and by the trading costs entailed by any re-hedging:

$$
W_{t+1}=\left[1+\rho_{t}\left(u_{t}-1\right)-\Delta F_{\gamma}\left(\Delta \chi_{t}\right)\right] W_{t}
$$


Most generally trading costs are described by a semi-positive definite function $\Delta F_{\gamma}$ vanishing only if the investor remains idle, i.e. when $\Delta \chi$ is zero.

From (49), (50) the variation of the invested capital fraction $\rho_{t}$ over a time unit is

$$
\Delta \rho_{t}=\frac{\rho_{t}+\left(u_{t}-1\right) \rho_{t}+\Delta \chi_{t}}{1+\rho_{t}\left(u_{t}-1\right)-\Delta F_{\gamma}\left(\Delta \chi_{t}\right)}-\rho_{t}
$$

The continuum limit is attained by replacing

$$
\begin{aligned}
& u_{t}-1 \rightarrow \mu d t+\sigma d B_{t} \\
& \Delta \chi_{t} \rightarrow f d t \\
& \Delta F \rightarrow \gamma \mathcal{F}(f) d t
\end{aligned}
$$

The differential $d u_{t}$ gives the relative stock price

$$
d u_{t}:=\frac{d p_{t}}{p_{t}}=\mu d t+\sigma d B_{t}
$$

The stochastic differential equation is defined according to the Ito convention. It has the solution

$$
p_{t}=p_{o} e^{\left(\mu-\frac{\sigma^{2}}{2}\right) t+\sigma B_{t}}
$$

A value of $\frac{\mu}{\sigma^{2}}$ outside the interval $[0,1]$ thus corresponds to strong inflation or deflation rates. If borrowing and short-selling is not allowed, the optimal strategy would then simply be to keep all money in stock or all money in bonds. If borrowing and short-selling is allowed, the problem becomes again similar to the one studied here, but the relevant intervals would then either be $[1, \infty]$ or $[-\infty, 0]$.

After a little algebra one finds

$$
\begin{aligned}
& d W_{t}=\mu \rho_{t} W_{t} d t+\sigma \rho_{t} W_{t} d B_{t}-\gamma W_{t} \mathcal{F} d t \\
& d \rho_{t}=\left[f+\rho_{t}\left(1-\rho_{t}\right)\left(\mu-\sigma^{2} \rho_{t}\right)\right] d t+\left[\sigma \rho_{t}\left(1-\rho_{t}\right)\right] d B_{t}+\gamma \rho_{t} \mathcal{F} d t(56)
\end{aligned}
$$

Equations (11) and (2) are recovered by setting

$$
\mathcal{F}=f^{2}
$$

The optimal control diverges in the limit of zero transaction costs so to hold the fraction in stocks tightly to the constant value $\mu / \sigma^{2}$. 


\section{References}

[1] K.K. Aase, "Optimum portfolio diversification in a general continuous-time model”, Stoch. Proc. and their Applications 18, (1984) 81-88.

[2] E. Aurell, R. Baviera, O. Hammarlid, M. Serva and A. Vulpiani, "A general methodology to price and hedge derivatives in incomplete markets", International J. of Theoretical and Applied Finance (IJTAF) 3, No. 1, (2000) 1-25 and http://xxx.lanl.gov/abs/cond-mat/9810257.

[3] E. Aurell and P. Muratore-Ginanneschi, "Financial Friction and Multiplicative Markov Market Game", International J. of Theoretical and Applied Finance (IJTAF) 3, No. 3 (2000) 501-510 and http://xxx.lanl.gov/abs/cond-mat/9908253.

[4] A. Borodin, P. Salminen Handbook of Brownian Motion (Birkhauser Verlag 2002).

[5] L.I. Chen, N.D. Goldenfeld and Y. Oono, "The renormalization group and singular perturbations: multiple-scales, boundary layers and reductive perturbation theory" Phys. Rev. E 54, 376-394 (1996).

[6] T. Cover, "Universal Portfolios", Mathematical Finance, 1, 1-29 (1991).

[7] T. Cover and E. Ordentlich, "Universal Portfolios with Side Information", IEEE Transactions on Information Theory, 42, 348-363 (1996).

[8] I. Estigneev and K.R. Schenk-Hoppé, "From rags to riches: on constant proportion investment strategies", International J. of Theoretical and Applied Finance (IJTAF) 5, No. 6, (2002) 563-573.

[9] J.D. Farmer "Market force, ecology and evolution" Santa Fe Institute series Research in Economics 98-12-117e (1998) and http://www.santafe.edu/sfi/publications/Working-Papers.

[10] S. Fauve "Patterns in Fluid Flow" Woods Hole Oceanog. Inst. Tech. Rept., WHOI-92-16, (1991).

[11] W.H. Fleming and H. Mete Soner Controlled Markov Processes and Viscosity Solutions, (Springer-Verlag, Berlin 1992).

[12] U. Frisch Turbulence: The legacy of A. N. Kolmogorov (Cambridge University Press, 1995)

[13] N. Hakanson and W. Ziemba, "Capital Growth Theory", in Handbooks in $O R \& M S$, Vol.9, eds. R. Jarrow et al. (Elsevier Science, 1995).

[14] J.L. Kelly Jr., "A new interpretation of the Information Rate", Bell Syst. Tech. J. 35 (1956) 917. 
[15] S. Maslov and Y.-C. Zhang, "Optimal Investment Strategy for Risky Assets", International J. of Theoretical and Applied Finance 1 (1998) 377-388.

[16] B. Øksendal Stochastic Differential Equations, 5th ed. (Springer-Verlag, Berlin 1998).

[17] V.I. Oseledec, "A multiplicative ergodic theorem: Lyapunov characteristic numbers for dynamical systems", Trans. Moscow Math. Soc. 19 (1968) 197.

[18] J.J. Sakurai Modern Quantum Mechanics rev. ed., (Addison-Wesley Publishing Company 1994). 